\title{
Magnetic anisotropy of singly Mn-doped InAs/GaAs quantum dots
}

\author{
Olivier Krebs, Emile Benjamin, and Aristide Lemaître \\ Laboratoire de Photonique et Nanostructures-CNRS, Route de Nozay, 91460 Marcoussis, France
}

(Received 3 September 2009; published 9 October 2009)

\begin{abstract}
We report on the microphotoluminescence spectroscopy of InAs/GaAs quantum dots (QDs) doped by a single $\mathrm{Mn}$ atom in a magnetic field either longitudinal or perpendicular to the optical axis. In both cases the spectral features of positive trion $\left(X^{+}\right)$are found to split into strongly circularly polarized components, an effect very surprising in a perpendicular magnetic field. The field-induced splitting is ascribed to the transverse Zeeman splitting of the neutral acceptor complex $A^{0}$ issued by the $\mathrm{Mn}$ impurity, whereas the circular optical selection rules result from the $p$ - $d$ exchange which acts as a very strong longitudinal magnetic field inhibiting the spin mixing by the transverse field of the QD heavy-hole ground state. A theoretical model of the spin interactions which includes (i) the local strain anisotropy experienced by the acceptor level and (ii) the anisotropic exchange due to the out-of-center Mn position provides a very good agreement with our observations.
\end{abstract}

DOI: 10.1103/PhysRevB.80.165315

PACS number(s): 72.25.Fe, 71.35.Pq, 72.25.Rb, 78.67.Hc

Doping a semiconductor quantum dot (QD) with a single $\mathrm{Mn}$ atom brings up remarkable spin-related properties due to the $s p$ - $d$ exchange interactions between the confined carriers (electron and hole) and the magnetic impurity. In the last few years Mn-doped CdTe QD's have been extensively studied by microphotoluminescence ( $\mu$-PL) spectroscopy in an external magnetic field. ${ }^{1-4}$ Most of the observations were very well interpreted by assuming a 5/2 spin for the Mn ion acting on the carriers confined in a quantum dot through Heisenberg Hamiltonians. Yet, the strong vertical confinement of QD's along their growth axis, as well as their in-plane biaxial strain were shown to modify significantly the spectral features because of the resulting heavy-hole nature of the valence-band ground state.

The quite specific signature of InAs/GaAs quantum dots doped with a single $\mathrm{Mn}$ atom has been recently uncovered in $\mu$-PL spectroscopy. ${ }^{5}$ In this system, the Mn impurity acts as an effective $J=1$ spin with a noticeable fine-structure splitting in zero magnetic field. This results from the neutral acceptor $\left(A^{0}\right)$ complex formed by $\mathrm{Mn}$ in a III-V matrix, namely, a negatively charged center $A^{-}$and a bound hole $h_{1}{ }^{6-9}$ The $J=1$ spin corresponds to the ground state of the $3 d^{5} \mathrm{Mn}$ spin $S=5 / 2$ and the bound hole total angular momentum $J_{h_{1}}=3 / 2$ which interact via the antiferromagnetic $p$ - $d$ exchange. Its zero-field splitting results from some local anisotropy of the potential experienced by the bound hole. ${ }^{5,10,11}$ Within this interpretation, the anisotropy of the $A^{0}$ complex does not affect the optical selection rules of the QD interband transitions which still involve a conduction electron $(e)$ and a valence-band hole $\left(h_{2}\right)$ essentially of heavyhole character both with $S$-like orbital. This was shown in Ref. 5 where a longitudinal magnetic field split all the optical transitions into their circularly polarized $\left(\sigma^{ \pm}\right)$components. In this paper, we show that the optical selection rules in a transverse magnetic field are in contrast deeply affected by the anisotropy of the $A^{0}$ effective spin, besides in a rather nonintuitive way. Indeed, in a magnetic field perpendicular to the optical axis, the optical transitions which are expected to be linearly polarized as usually encountered in undoped InAs QDs, ${ }^{12-14}$ exhibit for Mn-doped InAs QDs a strong circular polarization $\left(\sigma^{+}\right.$or $\left.\sigma^{-}\right)$. We show that this effect results from the $A^{0}$ spin anisotropy which enables to split by Zeeman effect the ferromagnetic (FM) and antiferromagnetic (AFM) configurations of the $h_{2}-A^{0}$ complex, while the heavy-hole $h_{2}$ keeps a well-defined pseudospin $\Uparrow\left(J_{h_{2}, z}=+3 / 2\right)$ or $\Downarrow\left(J_{h_{2}, z}=-3 / 2\right)$.

We studied a sample grown by molecular-beam epitaxy on a semi-insulating GaAs [001] substrate which consists of a single layer of InAs/GaAs QD's randomly doped by a single $\mathrm{Mn}$ atom (see Ref. 5 for details). We estimate that $\sim 0.1-1 \%$ of the quantum dots are effectively doped by a single $\mathrm{Mn}$ atom. The $\mu$-PL spectroscopy of individual Mndoped InAs QDs was carried out with a split-coil magnetooptic cryostat. A $2 \mathrm{~mm}$ focal-length aspheric lens (NA 0.5) actuated by piezo motors was used to focus the He-Ne excitation laser and to collect the PL from the sample. This compact microscope, which integrates both the sample and the optical lens, can be rotated about the vertical axis of the cryostat in order to change the magnetic field direction with respect to the optical axis from parallel (Faraday configuration) to perpendicular (Voigt configuration). Relying on in situ sample imaging we could therefore study the same quantum dot in both configurations. All measurements presented here were performed at low temperature $(T=2 \mathrm{~K})$. The collected PL was dispersed by a $0.6 \mathrm{~m}$ focal-length double spectrometer and detected by a nitrogen-cooled CCD array camera.

Figures 1 and 2 report on the optical spectroscopy of a charged exciton $X^{+}$in the same Mn-doped InAs QD (QD1) measured, respectively, in Faraday and Voigt configuration. Let us first comment on the results shown in Fig. 1. As evidenced in Ref. 5, the Mn-doped InAs QD is identified by its spectral features in zero magnetic field shown in Fig. 1(b). It consists of two doublets separated by the exchange energy $\Delta$ between the FM and AFM configurations of $h_{2}-A^{0}$, plus a weaker line denoted $O$ which corresponds to the transition involving the $A^{0}$ state $J_{z}=0$. Another specific feature is the equal splitting $\delta$ of both FM and AFM doublets which is ascribed to the fine structure of $A^{0}$ in its anisotropic environment. 
(a)

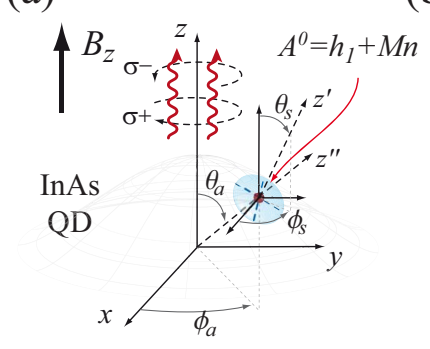

(b)

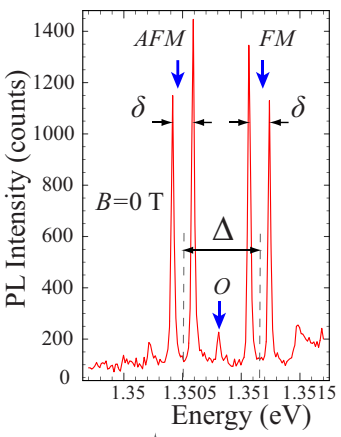

(c)
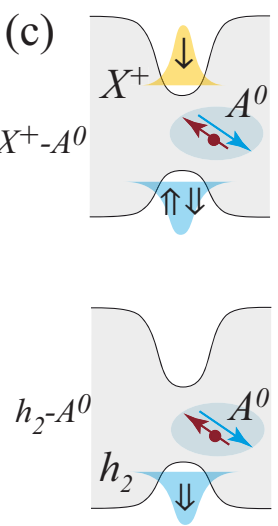

(d) Experience

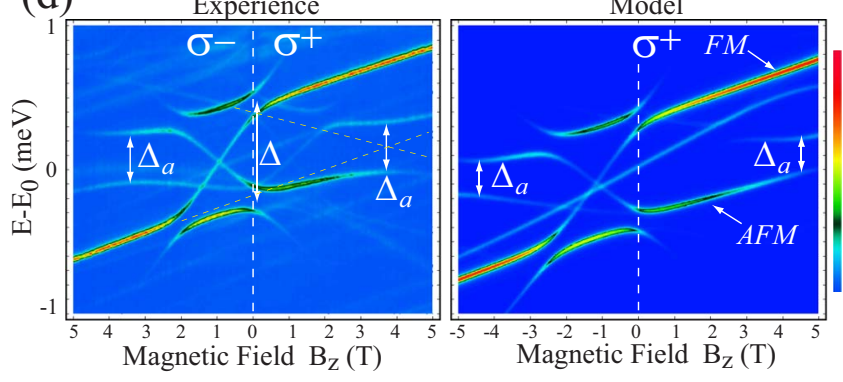

FIG. 1. (Color online) QD1 (a) schematics of an InAs QD doped by a single Mn atom forming a neutral acceptor $A^{0}$. (b) PL spectrum of a charged exciton $X^{+}$in such a QD at zero magnetic field. (c) Diagram of energy levels involved in the $X^{+}-A^{0} \rightarrow h_{2}-A^{0}$ transition. Solid (dashed) lines correspond to the levels with $\downarrow(\uparrow) e$ spin and $\Downarrow(\Uparrow) h_{2}$ pseudospin connected via a $\sigma^{+}\left(\sigma^{-}\right)$photon. (d) Contour plot of QD1 PL around $E_{0}=1.355 \mathrm{eV}$ as a function of longitudinal magnetic field measured in $\sigma^{-}$and $\sigma^{+}$circular polarizations (left) and theoretical simulation (right). The diamagnetic shift is subtracted so that the linear dependence on $B_{z}$ due to the Zeeman effect produces straight lines.

When the magnetic field is applied parallel to the optical axis $z$ the quantum levels involved in these optical transitions are split due to the Zeeman effect [see Fig. 1(c)], which gives rise to a very distinctive contour plot of the PL intensity as shown in Fig. 1(d). This "magneto-PL" image is composed of a series of spectra measured in both $\sigma^{+}$and $\sigma^{-}$polarizations, while the magnetic field was changed step by step with an increment of $50 \mathrm{mT}$ from 0 to $5 \mathrm{~T}$. The PL intensity is plotted against a linear color scale, and interpolation was used for a better graphical rendering. We observe a characteristic crosslike pattern, quite similar to the one reported in Ref. 5 for a negatively charged exciton $\left(X^{-}\right)$, with yet a no-
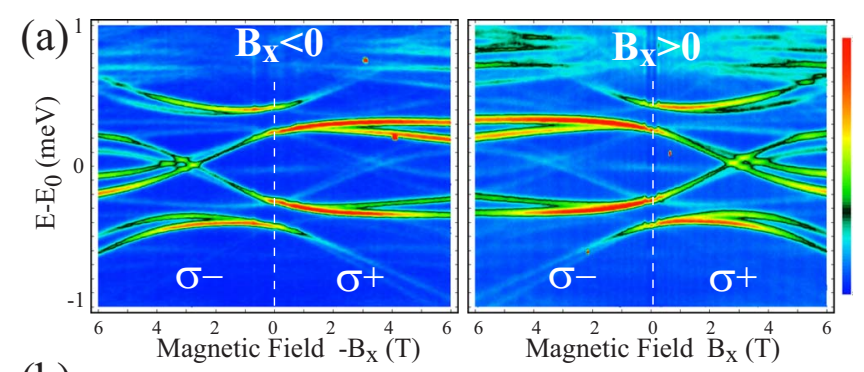

(b)
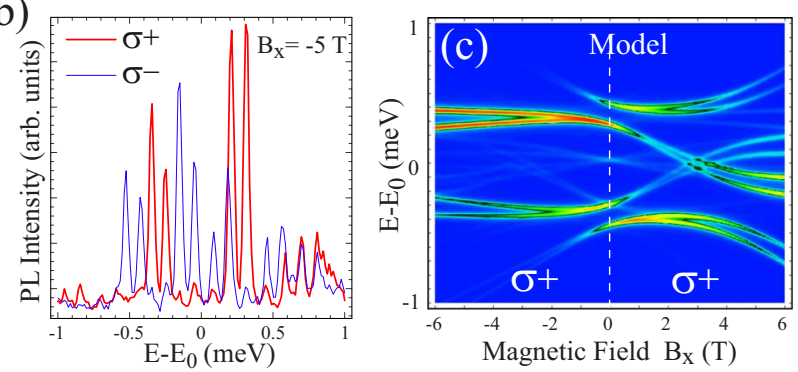

FIG. 2. (Color online) QD1 (a) PL Contour plot of the same $X^{+}$ of Fig. 1 as a function of transverse magnetic field $B_{x}$ positive (right) or negative (left). In both cases, the PL has been measured in $\sigma^{-}$and $\sigma^{+}$circular polarizations as indicated. The diamagnetic shift (half of that in Faraday configuration) has been subtracted. (b) PL spectra at $B_{x}=-5 \mathrm{~T}$ in both $\sigma^{+}$and $\sigma^{-}$polarizations. (c) Theoretical simulation of the PL contour plot (see text) with the same parameters as in Fig. 1.

ticeable difference. Here the cross shows up in negative fields (i.e., in $\sigma^{-}$polarization) while it was previously found in positive fields. This kind of mirror symmetry is similar to that observed in Refs. 2 and 5 between an exciton and a biexciton. Here, it corresponds to the symmetry between a positive trion $\left(X^{+}\right)$and a negative trion $\left(X^{-}\right)$. Indeed, both involve the same spin configurations either in the final or initial state of the optical recombination with yet orthogonal selection rules due to the Pauli principle. (For example, for $\left.\left.X^{+}|\Uparrow \downarrow \uparrow\rangle^{\sigma-} \rightarrow \Uparrow\right\rangle\right\rangle$ while for $X^{-}|\uparrow \downarrow \Uparrow\rangle^{\sigma+} \rightarrow|\uparrow\rangle$.) Note that in this sample most of the quantum dots (with or without Mn doping) were found as positively charged by an excess hole, after measuring the sign of the Overhauser shift (namely, the sign of nuclear polarization) generated under quasiresonant circularly polarized excitation. ${ }^{15,16}$

The $A^{0}$ exchange anisotropy originates from the position of the Mn atom out of the QD center. One can distinguish two main contributions: (i) the anisotropy of the local potential experienced by the $h_{1}$ bound hole which lifts the degeneracy of the $J=1$ state of $A^{0}$ (Refs. 5, 7, and 9) and (ii) the anisotropic part of the exchange coupling between the $h_{2}$ hole and the out-of-center Mn spin. ${ }^{17}$ The first contribution is responsible for the coupling of the $\left|J_{z}= \pm 1\right\rangle A^{0}$ states which gives rise to their anticrossing $\delta$ when they are brought into coincidence. This occurs in zero magnetic field for the $X^{+}-A^{0} \equiv e-A^{0}$ levels because the effective exchange with $X^{+}$ (namely, with a single $e$ spin) is essentially zero here, and at $B_{z} \approx \pm 2 \mathrm{~T}$ for the $h_{2}-A^{0}$ levels [see Fig. 1(c)]. More generally, this anisotropy is also responsible for the forbidden transitions corresponding to $\left|\Delta J_{z}\right|=2$ which form the cross pattern and which are partially permitted because $J_{z}$ is not a 
good quantum number. The second contribution is responsible for the weaker lines in Fig. 1(d) which gives rise to the anticrossings denoted $\Delta_{a}$. As discussed below, they result from a mixing of the $|\Uparrow, \pm 1\rangle$ and $|\Downarrow, \pm 1\rangle h_{2}-A^{0}$ states which makes visible the normally forbidden transitions $|\uparrow, \pm 1\rangle \rightarrow|\Downarrow, \pm 1\rangle$. Note however that all the lines keep a very strong circular polarization, because the $e-A^{0}$ initial states still have pure electron spin $\uparrow$ or $\downarrow$.

In Voigt configuration, the $A^{0}$ exchange anisotropy gives rise to a very surprising signature. When the transverse magnetic field is increased each line originating from the zerofield doublets splits into four lines with a strong $\sigma^{+}$or $\sigma^{-}$ circular polarization as reported in Fig. 2(b). Such a polarization is quite unexpected because, due to the mixing of the spin states for both the electron and hole by the field, the optical transitions of trions should become linearly polarized as observed in nonmagnetic QDs. ${ }^{12-14,18}$ Note the timereversal symmetry is yet well respected here since changing the sign of the applied magnetic field reverses the circular polarization from $\sigma^{ \pm}$to $\sigma^{\mp}$ [see Fig. 2(a)]. This clearly implies that the PL spectra depend on the magnetic field direction in the $x y$ plane because changing the sign of $B_{x}$ can be achieved by a $\pi$ rotation about $z$, which points out the role of $A^{0}$ in-plane anisotropy.

We have modeled these magneto-PL images by considering the spin Hamiltonians of the four involved particles (Mn, $h_{1}, e$, and $h_{2}$ ) assumed to occupy the ground state of their respective confinement potential. $e$ and $h_{2}$ have essentially the $S$-like character of the respective conduction and valence QD ground state, while $h_{1}$ is assumed to be strongly localized onto the Mn site which lies itself at a certain distance from the QD center [see Fig. 1(a)]. The theoretical indiscernibility between both overlapping holes is phenomenologically treated by introducing an exchange Hamiltonian between their spins (see below), which amounts to treat this issue in the Heitler-London scheme. ${ }^{19}$ This approach enables us to restrict ourselves to the spin degree of freedom of the four involved particles $\left(S_{\mathrm{Mn}}=5 / 2, J_{h_{1}}=3 / 2, S_{e}=1 / 2\right.$, and $J_{h_{2}}=3 / 2$ ), with interactions described using only their respective spin operators. The single-particle Hamiltonians read

$$
\begin{gathered}
\hat{\mathcal{H}}_{\mathrm{Mn}}=g_{\mathrm{Mn}} \mu_{\mathrm{B}} \hat{\boldsymbol{S}}_{\mathrm{Mn}} \cdot \boldsymbol{B}, \\
\hat{\mathcal{H}}_{h_{1}}=g_{h_{1}} \mu_{\mathrm{B}} \hat{\boldsymbol{J}}_{h_{1}} \cdot \boldsymbol{B}+\hat{\mathcal{H}}_{s}, \\
\hat{\mathcal{H}}_{h_{2}}=g_{h} \mu_{\mathrm{B}} \hat{\boldsymbol{J}}_{h_{2}} \cdot \boldsymbol{B}+\hat{\mathcal{H}}_{H L}, \\
\hat{\mathcal{H}}_{e}=g_{e} \mu_{\mathrm{B}} \hat{\boldsymbol{S}}_{e} \cdot \boldsymbol{B},
\end{gathered}
$$

where $\mu_{\mathrm{B}}$ is the Bohr magneton and $g_{\alpha}$ denotes the Landé factor of the particle $\alpha$ taken to the first order as a scalar for Mn, $h_{1}$ and $h_{2}$, while for $e$ a longitudinal $\left(g_{e, z}\right)$ and transverse $\left(g_{e, \perp}\right)$ factor will be used. In this formalism, the well-known anisotropy of the Landé factor for the hole states will naturally result from their splitting into heavy- and light-hole states. The potential anisotropy $\hat{\mathcal{H}}_{s}$ experienced by $h_{1}$ can be described via an effective strain tensor with three main axes that, in general, differ from the crystallographic axes. ${ }^{20}$ In that aim, we introduced as the dominant term a compressive strain $\epsilon_{\|}$along the $z^{\prime}$ direction defined by two angles $\theta_{s}$ and $\phi_{s}$ [see Fig. 1(a)] plus an in-plane shear strain $\epsilon_{\perp}$ with $x^{\prime}$ and $y^{\prime}$ main axes defined by an angle $\psi_{s}$ in the plane perpendicular to $z^{\prime} . \hat{\mathcal{H}}_{s}$ reads then

$$
\hat{\mathcal{H}}_{s}=-\frac{\epsilon_{\|}}{3}\left[\hat{J}_{h_{1}, z^{\prime}}^{2}-\frac{1}{2}\left(\hat{J}_{h_{1}, x^{\prime}}^{2}+\hat{J}_{h_{1}, y^{\prime}}^{2}\right)\right]+\frac{\epsilon_{\perp}}{2}\left(\hat{J}_{h_{1}, x^{\prime}}^{2}-\hat{J}_{h_{1}, y^{\prime}}^{2}\right),
$$

where the $\hat{J}_{h_{1}, \alpha^{\prime}}^{2}$ 's deduce from the $\hat{J}_{h_{1}, \alpha}^{2}$ operators by the three successive rotations transforming $z$ into $z^{\prime}$, namely, via the passage matrix $e^{-i \psi_{s} \hat{J}_{h_{1}}, z} e^{-i \hat{\theta}_{S} \hat{J}_{h_{1}, y}} e^{-i \phi_{s} \hat{J}_{h_{1}, z}}$. For the $h_{2}$ hole confined in the QD, we used a similar description, with $\Delta_{H L} \sim 30 \mathrm{meV}$ the splitting between the heavy-hole and light-hole states due to the stronger confinement along $z$. The corresponding term reads

$$
\hat{\mathcal{H}}_{H L}=-\frac{\Delta_{H L}}{3}\left[\hat{J}_{h_{2}, z}^{2}-\frac{1}{2}\left(\hat{J}_{h_{2}, x}^{2}+\hat{J}_{h_{2}, y}^{2}\right)\right] .
$$

We assumed that the exchange interactions between the different spins take the form of Heisenberg Hamiltonians, ${ }^{8,11}$ with besides a specific anisotropic part $\mathcal{H}_{a}$ for the $p$ - $d$ exchange between the out-of-center $\mathrm{Mn}$ and $h_{2} \cdot{ }^{17}$ Since the $e-A^{0}$ exchange turns out to be negligible in our experimental observations, which could be due to weak overlap with the $A^{0}$ impurity, we only consider here the exchange interactions involving the $\mathrm{Mn}, h_{1}$ and $h_{2}$ spins. The corresponding exchange Hamiltonian reads

$$
\hat{\mathcal{H}}_{X}=\varepsilon_{\mathrm{Mn}-h_{1}} \hat{\boldsymbol{S}}_{\mathrm{Mn}} \cdot \hat{\boldsymbol{J}}_{h_{1}}+\varepsilon_{\mathrm{Mn}-h_{2}} \hat{\boldsymbol{S}}_{\mathrm{Mn}} \cdot \hat{\boldsymbol{J}}_{h_{2}}+\varepsilon_{h_{1}-h_{2}} \hat{\boldsymbol{J}}_{h_{1}} \cdot \hat{\boldsymbol{J}}_{h_{2}}+\hat{\mathcal{H}}_{a}
$$

where the two-spin exchange energies $\varepsilon_{\alpha-\alpha^{\prime}}$ are considered as fitting parameters since they depend on the actual overlap between the particles. Yet, we expect the Mn-hole exchange interaction to be antiferromagnetic as usually reported in literature. The anisotropic part $\hat{\mathcal{H}}_{a}$ in Eq. (4) has been derived in Ref. 17 in the case of a spherical quantum dot. To the first order, it depends linearly on a parameter $\rho$ which depends itself on the Mn position and vanishes when it lies at the QD center. Although our InAs quantum dots are lens-shaped with no well-defined center, we assumed here the same expression for $\hat{\mathcal{H}}_{a}$ as follows:

$$
\hat{\mathcal{H}}_{a}=\rho \varepsilon_{\mathrm{Mn}-h_{2}}\left(\hat{J}_{h_{2}, z^{\prime \prime}}^{2} \hat{\boldsymbol{S}}_{\mathrm{Mn}} \cdot \hat{\boldsymbol{J}}_{h_{2}}+\hat{\boldsymbol{S}}_{\mathrm{Mn}} \cdot \hat{\boldsymbol{J}}_{h_{2}} \hat{J}_{h_{2}, z^{\prime \prime}}^{2}\right),
$$

where the angular momentum operator $\hat{J}_{h_{2}, z^{\prime \prime}}$ along the direction $z^{\prime \prime}$ [defined by two angles $\theta_{a}$ and $\phi_{a}$, see Fig. 1(a)] refers to the position of the $\mathrm{Mn}$ atom with respect to some effective QD center, e.g., the maximum of the $h_{2} S$-like envelope function.

To calculate the theoretical PL emission spectra, the Hamiltonians of the initial and final states in $X^{+}$transition are first diagonalized. In practice we restricted ourselves to the first $A^{0}$ levels $J=1$ [as represented in Fig. 1(c)] and $J=2$, where $\hat{\boldsymbol{J}}=\hat{\boldsymbol{S}}_{\mathrm{Mn}}+\hat{\boldsymbol{J}}_{h_{1}}$. The PL intensity emitted in the transition 
TABLE I. Parameters used for theoretical simulations. Strains $\left(\epsilon_{\|}, \epsilon_{\perp}\right)$ and exchange energies $\left(\varepsilon_{\mathrm{Mn}-h_{1}}, \varepsilon_{\mathrm{Mn}-h_{2}}, \varepsilon_{h_{1}-h_{2}}\right)$ are given in $\mathrm{meV}$.

\begin{tabular}{ccccccc}
\hline \hline & $g_{\mathrm{Mn}}$ & $g_{h_{1}}$ & $g_{h_{2}}$ & $g_{e, z}$ & $g_{e, \perp}$ & $T_{\mathrm{FS}}(K)$ \\
\hline & 2 & 0.8 & 0.85 & -0.6 & -0.35 & 15 \\
& & & & & & \\
& $\epsilon_{\|}$ & $\epsilon_{\perp}$ & $\theta_{s}(\mathrm{deg})$ & $\phi_{s}(\mathrm{deg})$ & $\psi_{s}(\mathrm{deg})$ & \\
\hline QD1 & 6.95 & 2.27 & $36^{\circ}$ & $-120^{\circ}$ & $49^{\circ}$ & \\
QD2 & 6.75 & 2.55 & $35^{\circ}$ & $-93^{\circ}$ & $55^{\circ}$ & \\
QD3 & 3.6 & 2 & $50^{\circ}$ & $55^{\circ}$ & $40^{\circ}$ & \\
& & & & & & \\
& $\varepsilon_{\mathrm{Mn}-h_{1}}$ & $\varepsilon_{\mathrm{Mn}-h_{2}}$ & $\varepsilon_{h_{1}-h_{2}}$ & $\rho$ & $\theta_{a}(\mathrm{deg})$ & $\phi_{a}(\mathrm{deg})$ \\
\hline QD1 & 4.2 & 0.6 & 1.2 & -0.1 & $81^{\circ}$ & $-120^{\circ}$ \\
QD2 & 4.5 & 0.63 & 1.35 & -0.19 & $85^{\circ}$ & $-123^{\circ}$ \\
QD3 & 5 & 0.85 & 1.8 & -0.12 & $102^{\circ}$ & $55^{\circ}$ \\
\hline \hline
\end{tabular}

from $|i\rangle$ to $|f\rangle$ is calculated by taking into account both the oscillator strength $\propto\left|\left\langle f\left|\hat{P}_{\sigma^{+}}\right| i\right\rangle\right|^{2}$ (where $\hat{P}_{\sigma^{+}}$is the dipolar operator for a $\sigma^{+}$polarization) and the population $\rho_{i i}$ of the initial state $|i\rangle$. As He-Ne excitation produces nonpolarized carriers and electron spin relaxation may be considered negligible in QDs during the radiative lifetime of $X^{+}(\approx 1 \mathrm{~ns})$, the $\rho_{i i}$ 's are determined only by the populations in the different states of $A^{0}$. Assuming that under weak optical excitation density, thermalization occurs mostly in the final state (FS) of the system (here the $h_{2}-A^{0}$ complex) to a certain equilibrium temperature $T_{\mathrm{FS}}$, we deduced the $\rho_{i i}$ 's from the partial trace of the FS density matrix $\rho_{\mathrm{FS}}=\exp \left(-\hat{H}_{\mathrm{FS}} / k_{\mathrm{B}} T_{\mathrm{FS}}\right) / \operatorname{Tr}\left[\exp \left(-\hat{H}_{\mathrm{FS}} / k_{\mathrm{B}} T_{\mathrm{FS}}\right)\right]$, where $k_{\mathrm{B}}$ is the Boltzmann factor. Eventually, to compare with the experiments we applied to the calculated transitions a Lorentzian broadening of FWHM $=25 \mu \mathrm{eV}$.

The above model enabled us to reproduce quite well both magneto-PL images performed in Faraday and Voigt configurations by using the same set of parameters (see Table I). The electron and hole Landé factors were determined in order to reproduce the linear slopes due to the Zeeman effect. We found very similar values for the three quantum dots investigated here. Remarkably, thanks to the strong exchange field experienced by $h_{2}$, the transverse electron Landé factor $g_{e, \perp}$ could be determined unambiguously from the common splittings $(\sim 125 \mu \mathrm{eV}$ at $6 \mathrm{~T})$ of the transitions measured in circular polarization, see Fig. 2. The parameters describing the local anisotropy [Eq. (2)], the exchange strength [Eq. (4)], and the $A^{0}$ position anisotropy [Eq. (5)] were manually adjusted to reproduce the most remarkable features of the experimental images: e.g., the zero-field anticrossing $\delta$, the FM-AFM exchange $\Delta$, the anticrossing $\Delta_{a}$ or the $\sigma^{+} / \sigma^{-}$Zeeman splitting in Voigt configuration. In contrast to our previous assumption in Ref. 5 and to the geometrical effects discussed in the case of II-VI QDs, ${ }^{4}$ we did not include any heavy-hole light-hole mixing due to QD in-plane asymmetry to reproduce the anticrossing $\Delta_{a}$. Actually, the latter can be fully ascribed to the $A^{0}$ position anisotropy, since
$\Delta_{a} \propto \rho \varepsilon_{\mathrm{Mn}-h_{2}} \sin ^{2} \theta_{a}$. The heavy-light hole splitting $\Delta_{H L}$ was assumed to amount to a few $10 \mathrm{meV}$, so that the $h_{2}$ hole has a dominant heavy-hole character in agreement with the PL polarization in Fig. 1. ${ }^{21}$ Besides, the strong circular polarization still measured in Voigt [up to 90\%, see Fig. 2(b)] indicates that the $h_{2}$ hole spin states $|\Uparrow\rangle$ and $|\Downarrow\rangle$ are essentially not mixed by the magnetic field, in contrast to undoped InAs QD's where an effective transverse $g$-factor $g_{h_{\perp}} \sim 0.3$ is found. ${ }^{13,22}$ We ascribe this effect to the strong FM-AFM exchange $\Delta$ which inhibits this coupling for magnetic fields as long as $\left|B_{x}\right|<\Delta /\left(g_{h}, \mu_{B}\right) \sim 50 \mathrm{~T}$. We still have to explain the splitting between the $\sigma^{+}$and $\sigma^{-}$components, namely, between the $|\Uparrow, \pm 1\rangle$ and $|\Downarrow, \pm 1\rangle$ states. This effect which is well reproduced by our model [see Fig. 2(c)] results from the local anisotropy of $A^{0}$ which yields a finite in-plane spin projection of the $| \pm 1\rangle$ states. The latter experience therefore a Zeeman effect in a transverse magnetic field dragging along the almost pure hole spin states $\Uparrow$ and $\Downarrow$. The resulting splitting $\Delta_{\Uparrow, \Downarrow}$ of the FM and AFM $h_{2}-A^{0}$ levels calculated within second-order perturbation theory reads ${ }^{23}$

$$
\Delta_{\Uparrow, \Downarrow}=\frac{7 g_{\mathrm{Mn}}-3 g_{h_{1}}}{5} \frac{\cos \phi_{s} \sin 2 \theta_{s}\left(\epsilon_{\perp}+\epsilon_{\|}\right)}{6 \varepsilon_{h_{1}-h_{2}}-14 \varepsilon_{\mathrm{Mn}-h_{2}}} \mu_{\mathrm{B}} B_{x} .
$$

As logically expected, the splitting $\Delta_{\Uparrow, \Downarrow}$ vanishes for a magnetic field perpendicular to $z^{\prime}\left(\phi_{s}= \pm \pi / 2\right)$ or for $\theta_{s}=0$ or $\pi / 2$ when cylindrical symmetry is restored. Experimentally, we indeed observed a large variety of magneto-PL images in Voigt configuration when measuring different $\mathrm{Mn}$ doped InAs QDs. QD2 shown in Fig. 3 is very similar to QD1 in Faraday configuration but exhibits a very distinctive behavior in Voigt. The Voigt magneto-PL image is almost symmetrical between $\sigma^{+}$and $\sigma^{-}$polarizations, which means that individual lines are now weakly circularly polarized. Such a situation is expected when the main strain axis $z^{\prime}$ is almost orthogonal to the field direction. We could indeed reproduce QD2 magneto-PL images by taking $\phi_{s}=-93^{\circ}$, whereas other fitting parameters were found close to those of QD1. Note, however, that the anisotropic exchange parameter $\rho$ is almost twice that for QD1. This was found necessary to reproduce a third zero-field doublet [denoted $O^{\prime}$ in Fig. 3(a)] with the same $\delta$ splitting as the FM and AFM doublets. This doublet corresponds to the transition from the two $e-A^{0}$ lower states [split by $\delta$ in zero field, see Fig. 1(c)] to the $J_{z}=0 h_{2}-A^{0}$ level. This normally forbidden transition becomes permitted here thanks to the strong anisotropic exchange $\propto \rho$.

Remarkably, the relative position of these three doublets reflects thus directly the level fan chart of $h_{2}-A^{0}$ in zero field. Figure 4 illustrates another interesting case (QD3), where the strain anisotropy experienced by $h_{1}$ is no longer dominated by a strong uniaxial strain $\epsilon_{\|}$along $z^{\prime}$. As shown in Fig. 4(a), the zero-field spectrum of QD3 strongly deviates from our usual observation of Mn-doped QDs. Up to nine lines are visible, but no doublet structures can be clearly perceived. By performing the autocorrelation of these normalized peaks, we found that these lines perfectly reflect all $3 \times 3$ possible transitions from $e-A^{0}$ to $h_{2}-A^{0}$ as indicated by $i \bullet j$ labels $(i, j=1,2,3)$ in the figure. This is evidenced by the 

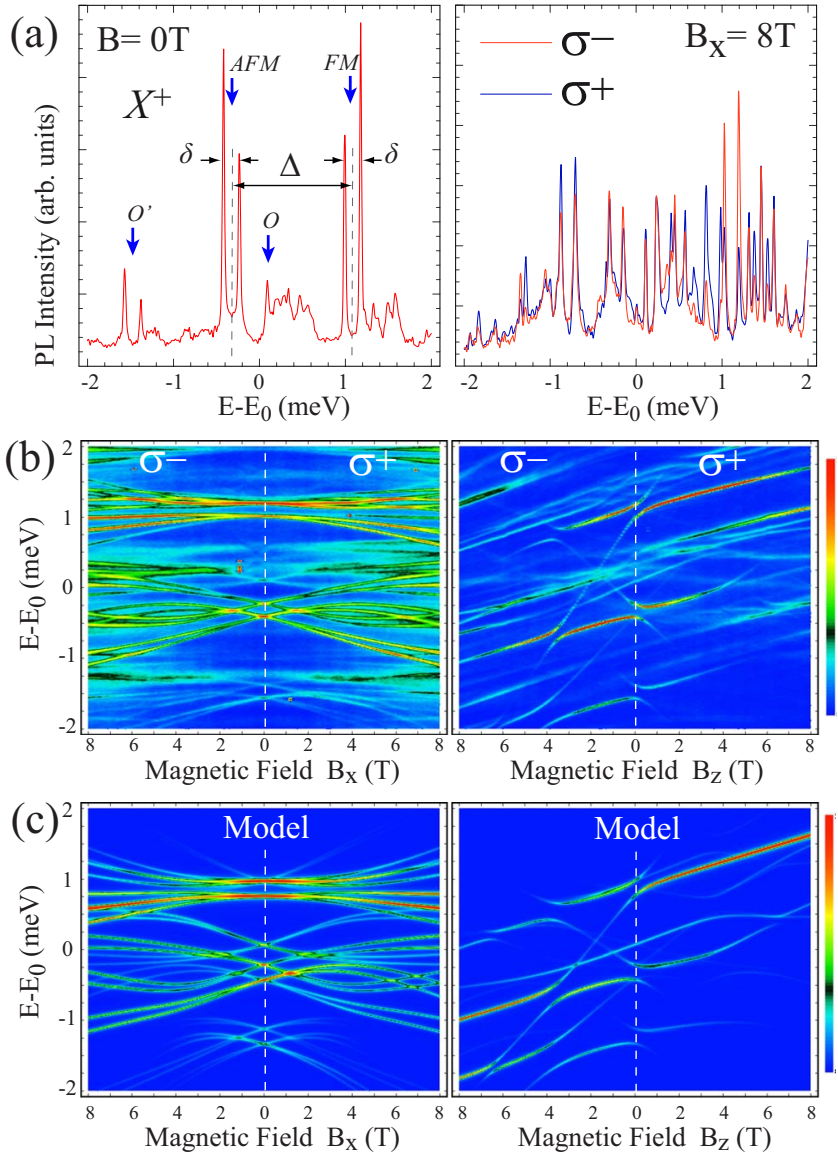

FIG. 3. (Color online) QD2. (a) PL spectra of an $X^{+}$trion at $B=0 \mathrm{~T}$ and $B_{x}=8 \mathrm{~T}$ centered at $E_{0}=1.355 \mathrm{eV}$. (b) PL Contour plot as a function of transverse (left) and longitudinal (right) magnetic fields in $\sigma^{-}$and $\sigma^{+}$circular polarizations as indicated. The diamagnetic shift is subtracted. Additional spectral features likely due to different charge states of QD2 are also visible (c) Theoretical simulation of the PL contour plot.

horizontal bars in Fig. 4(a) which show that all two-level splittings (in the initial or final shells) appear three times in the measured spectrum. The images in Voigt and Faraday showing both many anticrossings and nonlinear field dependencies confirm that these lines originate from the same Mndoped QD, even though the crosslike pattern usually observed is now hardly perceptible. Quite remarkably, our model enables us to reproduce still fairly well the experimental magneto-PL images. We essentially had to reduce the strength of $\epsilon_{\|}$(see Table I) while keeping the other parameters close to those of QD1. This indeed leads to eigenstates with $A^{0}$ angular momentum very different in the initial $\left(e-A^{0}\right)$ and final $\left(h_{2}-A^{0}\right)$ shells, so that all transitions become partially allowed. In Voigt configuration, we still observe, such as for QD1, a marked field-induced splitting due to the transverse Zeeman effect of the $A^{0}$ complex. However, the circular polarization of the transitions is now much weaker $(<\sim 30 \%)$ than for QD1. This is due to the mixing of the $h_{2}$ spin states by the anisotropic exchange $\hat{\mathcal{H}}_{a}$, which is appreciably enhanced with respect to QD1 because of the smaller strain-induced splittings of $A^{0}$ levels.

Our experimental observations and numerical simulations
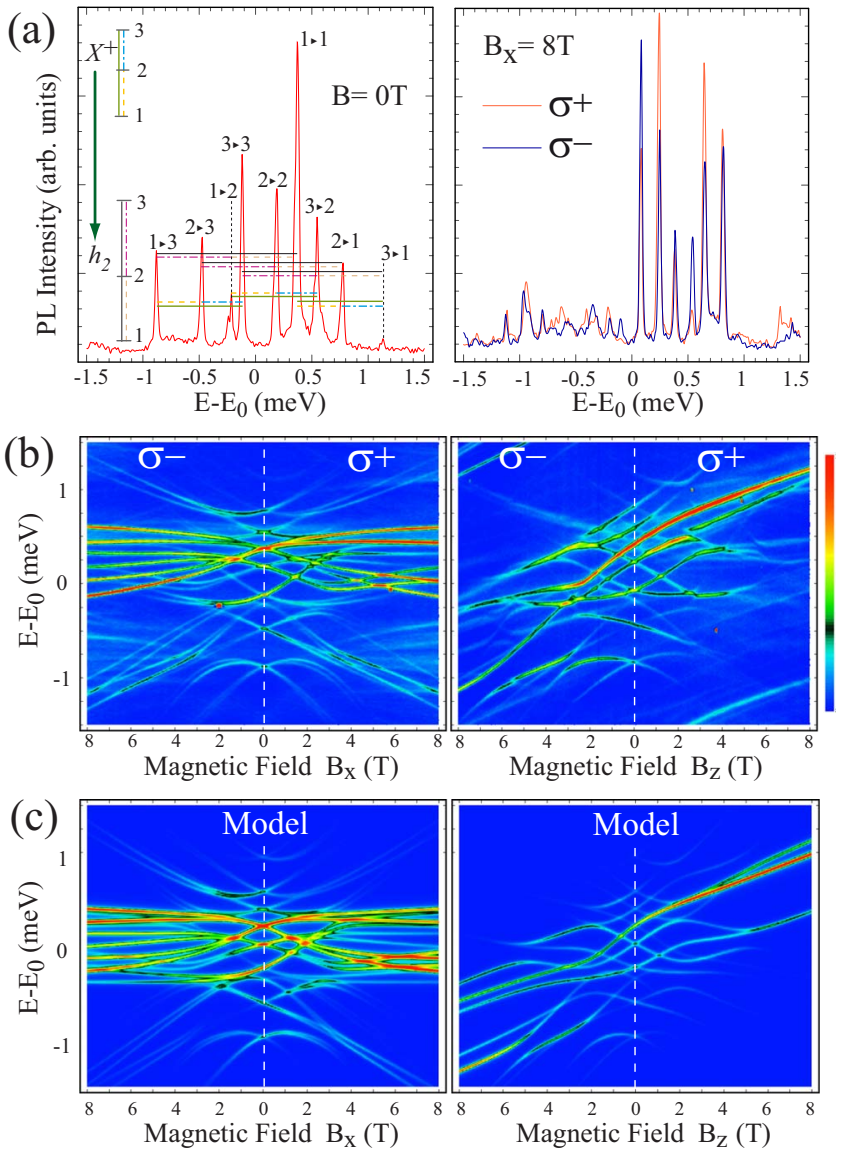

FIG. 4. (Color online) QD3 (a) PL spectra of an $X^{+}$trion at $B=0 \mathrm{~T}$ and $B_{x}=8 \mathrm{~T}$ centered at $E_{0}=1.3525 \mathrm{eV}$. Inset at zero field shows the $3 \times 3$ level fan chart responsible for the observed line splittings. (b) PL Contour plot as a function of transverse (left) and longitudinal (right) magnetic fields in $\sigma^{-}$and $\sigma^{+}$circular polarizations as indicated. The diamagnetic shift is subtracted. (c) Theoretical simulation of the PL contour plot.

indicate that so far we have likely studied Mn-doped InAs QDs with strongly uncentered $\mathrm{Mn}$ atom. This sounds compatible with the fundamental issue raised in Ref. 24 where a singlet configuration in a common $S$-like orbital is predicted for the $h_{1}-h_{2}$ ground state when the Mn atom lies exactly at the QD center. Our model describes instead two holes occupying distinct orbitals at different positions with a finite overlap between them giving rise to the AFM coupling as for electrons in the $\mathrm{H}_{2}$ molecule. ${ }^{19}$

In conclusion, the $\mu$-PL investigation in both a longitudinal and transverse magnetic field of individual singly $\mathrm{Mn}$ doped InAs QDs reveals remarkable insights into the spin interactions between carriers and a Mn impurity in a III-V matrix. The explicit anisotropic part to the $p-d$ exchange $^{17}$ due to the Mn position with respect to the QD center explains better than the QD geometrical anisotropy ${ }^{4}$ certain forbidden transitions and anticrossings observed in Faraday configuration. More spectacular is still the conservation of circularly polarized selection rules along with Zeeman splitting in a transverse magnetic field. This nonintuitive result is remarkably well interpreted by considering pure heavy-hole states in the quantum dot and local potential anisotropy ex- 
perienced by the acceptor level bound to the Mn impurity. The latter plays a crucial role to explain the dependence on the in-plane (azimuthal) magnetic field angle, as well as the effective optical selection rules. Our results which validate the picture of a Mn impurity keeping a tightly bound hole in spite of the QD strain and composition, ${ }^{24}$ opens the way toward resonant experiments similar to those recently achieved in semiconductor QD molecule ${ }^{25}$ in order to optically prepare and read out a single spin.

We acknowledge fruitful discussions with A. K. Bhattachargee and S. Chutia. This work was partly supported by the Région Ile-de-France and ANR contracts BOITQUANT and MOMES.
${ }^{1}$ L. Besombes, Y. Leger, L. Maingault, D. Ferrand, H. Mariette, and J. Cibert, Phys. Rev. Lett. 93, 207403 (2004).

${ }^{2}$ L. Besombes, Y. Leger, L. Maingault, D. Ferrand, H. Mariette, and J. Cibert, Phys. Rev. B 71, 161307(R) (2005).

${ }^{3}$ Y. Léger, L. Besombes, J. Fernández-Rossier, L. Maingault, and H. Mariette, Phys. Rev. Lett. 97, 107401 (2006).

${ }^{4}$ Y. Léger, L. Besombes, L. Maingault, D. Ferrand, and H. Mariette, Phys. Rev. Lett. 95, 047403 (2005).

${ }^{5}$ A. Kudelski, A. Lemaître, A. Miard, P. Voisin, T. C. M. Graham, R. J. Warburton, and O. Krebs, Phys. Rev. Lett. 99, 247209 (2007).

${ }^{6}$ J. Szczytko, W. Mac, A. Stachow, A. Twardowski, P. Becla, and J. Tworzydlo, Solid State Commun. 99, 927 (1996).

${ }^{7}$ J. Schneider, U. Kaufmann, W. Wilkening, M. Baeumler, and F. Köhl, Phys. Rev. Lett. 59, 240 (1987).

${ }^{8}$ A. K. Bhattacharjee and C. B. à la Guillaume, Solid State Commun. 113, 17 (1999).

${ }^{9}$ A. O. Govorov, Phys. Rev. B 70, 035321 (2004).

${ }^{10}$ J. van Bree, P. M. Koenraad, and J. Fernández-Rossier, Phys. Rev. B 78, 165414 (2008).

${ }^{11}$ A. O. Govorov, C. R. Phys. 9, 857 (2008).

${ }^{12}$ Y. Léger, L. Besombes, L. Maingault, and H. Mariette, Phys. Rev. B 76, 045331 (2007).

${ }^{13}$ X. Xu, Y. Wu, B. Sun, Q. Huang, J. Cheng, D. G. Steel, A. S. Bracker, D. Gammon, C. Emary, and L. J. Sham, Phys. Rev. Lett. 99, 097401 (2007).

${ }^{14}$ A. V. Koudinov, I. A. Akimov, Y. G. Kusrayev, and F. Henneberger, Phys. Rev. B 70, 241305(R) (2004).

${ }^{15}$ B. Eble, O. Krebs, A. Lemaître, K. Kowalik, A. Kudelski, P.
Voisin, B. Urbaszek, X. Marie, and T. Amand, Phys. Rev. B 74, 081306(R) (2006).

${ }^{16}$ O. Krebs, B. Eble, A. Lemaître, P. Voisin, B. Urbaszek, T. Amand, and X. Marie, C. R. Phys. 9, 874 (2008).

${ }^{17}$ A. K. Bhattacharjee, Phys. Rev. B 76, 075305 (2007).

${ }^{18}$ G. Fernandez, T. Volz, R. Desbuquois, A. Badolato, and A. Imamoglu, Phys. Rev. Lett. 103, 087406 (2009).

${ }^{19} \mathrm{P}$. Fazekas, Lecture Notes on Electron Correlation and Magnetism, Series in Modern Condensed Matter Physics Vol. 5 (World Scientific, Singapore, 1999).

${ }^{20}$ A. M. Yakunin, A. Yu. Silov, P. M. Koenraad, J.-M. Tang, M. E. Flatté, J.-L. Primus, W. Van Roy, J. De Boeck, A. M. Monakhov, K. S. Romanov, I. E. Panaiotti, and N. S. Averkiev, Nature Mater. 6, 512 (2007).

${ }^{21}$ This parameter cannot be determined with precision because it has basically no influence on the simulated spectra, as soon as it guarantees a clear separation between the heavy and light holes, namely, above $\sim 10 \mathrm{meV}$.

${ }^{22}$ M. Bayer, G. Ortner, O. Stern, A. Kuther, A. A. Gorbunov, A. Forchel, P. Hawrylak, S. Fafard, K. Hinzer, T. L. Reinecke, S. N. Walck, J. P. Reithmaier, F. Klopf, and F. Schäfer, Phys. Rev. B 65, 195315 (2002).

${ }^{23}$ To simplify the general expression we took $\psi_{s}=0$.

${ }^{24}$ S. Chutia and A. K. Bhattacharjee, Phys. Rev. B 78, 195311 (2008).

${ }^{25}$ D. Kim, S. E. Economou, Ştefan C. Bădescu, M. Scheibner, A. S. Bracker, M. Bashkansky, T. L. Reinecke, and D. Gammon, Phys. Rev. Lett. 101, 236804 (2008). 